\title{
Enseñanza-Aprendizaje en Robótica. Construcción de Simuladores como Actividades de Comprensión
}

\author{
Oscar D. Morán y Ricardo R. Monasterolo \\ Universidad Nacional de San Luis, Facultad de Ingeniería y Ciencias Económico Sociales, \\ Avda. 25 de Mayo N³84, (5730 )Villa Mercedes, San Luis-Argentina \\ (e-mail: dmoran@fices.unsl.edu.ar, monaster@fices.unsl.edu.ar)
}

\begin{abstract}
Resumen
El objetivo de este trabajo es aportar en el mejoramiento de los procesos de enseñanza y aprendizaje de la asignatura robótica, en el marco de la denominada "enseñanza para la comprensión". Esto con el objeto de facilitar la comprensión de los conceptos básicos del funcionamiento de los robots antropomorfos por parte de los alumnos, Para esto, se diseñó un dispositivo didáctico, orientado a que el alumno desarrolle un programa de simulación de complejidad creciente, que abarca desde el movimiento de una línea hasta la simulación de un robot con tres grados de libertad. Se presentan los niveles de comprensión alcanzados por los estudiantes utilizando el dispositivo diseñado. Se deduce que las características de las actividades de comprensión realizadas y el nivel exigido para aprobar la asignatura, aseguran que la mayoría de los estudiantes alcancen un buen nivel de comprensión de conceptos básicos y aplicados de robótica.
\end{abstract}

Palabras clave: robótica, simulación, enseñanza y aprendizaje, comprensión, didáctica

\section{Teaching-Learning in Robotics. Construction of Simulators as Activities of Understanding}

\begin{abstract}
The objective of the work is to contribute in improving the teaching and learning processes of a course on robotics, within the concept of "teaching for understanding". This is done to facilitate students understanding of the basic concepts of anthropomorphic robots operation. For this, a didactic device, designed in such a way that students develop a simulation program of increasing complexity, ranging from the movement of a line to the simulation of a robot whit three degrees of freedom was implemented. This paper also presents the levels of understanding achieved by students using the designed device. It was found that the characteristics of the activities done by the students and the level required to pass the course, ensure that most students achieve a good level of comprehension of basic and applied concepts on robotics.
\end{abstract}

Keywords: robotics, simulation, teaching and learning, understanding, didactics 


\section{INTRODUCCIÓN}

En la asignatura Robótica, de 5to año de las carreras de Ingeniería Electromecánica y Electrónica, de la Facultad de Ingeniería y Ciencias Económico - Sociales, de la Universidad Nacional de San Luis, se enseña el funcionamiento de los robots antropomorfos, principalmente enfocado al estudio del sistema de control de los mismos. Esto requiere la compleja tarea de enseñar como se mueven los ejes de los motores de las articulaciones, controlados numéricamente. Lograr que los alumnos se apropien de este conocimiento, que lo puedan usar con flexibilidad y más allá de lo que saben, es complicado.

Considerando que para comprender ideas complejas y formas de investigación, los estudiantes deben aprender haciendo, se diseñó un dispositivo didáctico basada en el marco teórico de la enseñanza para la comprensión, desarrollado en el Proyecto Cero de la Universidad de Harvard (Blythe, 1999). El mismo incluye actividades de comprensión que deben realizar los estudiantes, que consisten en la construcción de simuladores de complejidad creciente, hasta llegar al desarrollo del simulador de un robot con animación gráfica, utilizando el lenguaje de programación que viene incorporado en Matlab (Wiske et al., 2006).

Progresivamente el alumno construye el software que le permite realizar la simulación del movimiento de una línea en el espacio, luego desarrolla el programa de un simulador del problema cinemático directo, el simulador del problema cinemático inverso, para culminar con un trabajo integrador, que consiste en construir un simulador con animación gráfica del control cinemático de un robot antropomorfo de tres grados de libertad.

En el dispositivo didáctico diseñado en esta investigación, el alumno va construyendo el programa sentencia por sentencia profundizando en los conceptos ya que debe manipular todas las variables y utilizar los contenidos teóricos, para así construir el simulador, lo que favorece su comprensión. En el proceso de construir el programa de simulación utilizando un lenguaje básico de programación es donde el alumno va incorporando conceptos abstractos que de otra manera son difíciles de transmitir por parte del profesor y de comprender por parte del estudiante. Esta metodología se diferencia de las más difundidas, donde el profesor utiliza plataformas o software específicamente preparado, para montar una simulación. No se han encontrado antecedentes específicos, en el que se utilice la construcción de simuladores como actividades de comprensión, para la enseñanza del funcionamiento interno de un robot antropomorfo, al nivel del movimiento combinado de cada una de sus articulaciones, impulsadas por actuadores rotativos o lineales, que es lo que se estudia en este trabajo. Existen algunos ejemplos del uso de simuladores como un instrumento de apoyo para la enseñanza de la robótica (Herías et al., 2006; Bravo et al., 2007). Hay ejemplos del uso combinado de software y hardware de robots educacionales para la enseñanza (Alimisis et al., 2007; Rawat y Massiha, 2003). También existen paquetes de simuladores y/o entrenadores comerciales para enseñar a utilizar robots, o simuladores para analizar la integración de robots en medios productivos.

El estudio realizado en este trabajo, es de tipo exploratorio - descriptivo. Es exploratorio dado que no se han encontrado antecedentes publicados, sobre el diseño, implementación y evaluación de actividades de comprensión, basadas en la construcción de simuladores por parte de los alumnos, utilizando un lenguaje de programación, en un curso de robótica. Es descriptivo porque logra caracterizar los niveles de comprensión alcanzados por los estudiantes, en cada una de las dimensiones de la misma, en el contexto de la situación de enseñanza llevada a cabo en la asignatura utilizando el dispositivo diseñado.

\section{METODOLOGIA}

Con el objetivo de que el alumno "aprenda haciendo", se diseñaron una serie de actividades de comprensión a realizar por los alumnos, que se van concatenando de manera tal que la complejidad creciente de las mismas permiten lograr las metas parciales necesarias, hasta alcanzar la meta final, con la realización de un trabajo final integrador (TFI). El alumno debe realizar en forma individual cada trabajo práctico, con excepción del integrador que puede ser 
realizado por dos alumnos trabajando en equipo. Una síntesis de los trabajos prácticos a desarrollar como actividades de comprensión es la mostrada en la Tabla 1.

Hay tres actividades que se corresponden con los trabajos prácticos 4,5 y 6 , en las cuales el alumno, a partir de los conocimientos previos, debe obtener el modelo de Denavit - Hartenberg de un robot y desarrollar un software de simulación. Es importante destacar que en la construcción del simulador no se utilizan, plataformas, ni programas específicos preparados para realizar simulaciones, sino el lenguaje que viene incluido en Matlab.

Tabla 1: Síntesis de Trabajos Prácticos

\begin{tabular}{|c|c|c|}
\hline 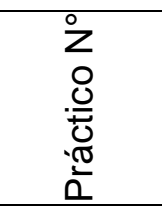 & ACTIVIDAD DE COMPRENSIÓN & METAS DE COMPRENSIÓN \\
\hline 1 & $\begin{array}{l}\text { Realizar operaciones de transformaciones } \\
\text { espaciales aplicando matrices de rotación } \\
3 \times 3 \text { utilizando Matlab }\end{array}$ & $\begin{array}{l}\text { Dominar las transformaciones } \\
\text { espaciales de rotación. } \\
\text { Obtener destreza en el uso de Matlab } \\
\text { para resolver este tipo de } \\
\text { operaciones. }\end{array}$ \\
\hline 2 & $\begin{array}{l}\text { Realizar operaciones de transformaciones } \\
\text { espaciales de traslación y rotación } \\
\text { combinadas. } \\
\text { Graficar vectores y mover líneas usando el } \\
\text { lenguaje de programación incorporado en } \\
\text { Matlab }\end{array}$ & $\begin{array}{l}\text { Dominar las transformaciones } \\
\text { espaciales de traslación y de rotación } \\
\text { combinadas. } \\
\text { Animar objetos gráficos con Matlab. } \\
\text { Obtener destreza en el uso de } \\
\text { Matlab. }\end{array}$ \\
\hline 3 & $\begin{array}{l}\text { Obtención de modelos para distintas } \\
\text { configuraciones de robots aplicando la } \\
\text { metodología sistemática de Denavit- } \\
\text { Hartenberg (Barrientos, 1997). }\end{array}$ & $\begin{array}{l}\text { Obtener modelos de robots, en forma } \\
\text { metódica y sistemática. }\end{array}$ \\
\hline 4 & $\begin{array}{l}\text { Obtención de la cinemática directa (CD) } \\
\text { para distintas configuraciones de robots } \\
\text { (Barrientos, 1997). } \\
\text { Construcción de un software para simular la } \\
\text { CD con animación gráfica, con ingreso de } \\
\text { datos por teclado, para cada una de las } \\
\text { configuraciones propuestas. }\end{array}$ & $\begin{array}{l}\text { Comprender la solución de la } \\
\text { cinemática directa de un robot. }\end{array}$ \\
\hline 5 & $\begin{array}{l}\text { Obtención de la cinemática inversa }(\mathrm{Cl}) \\
\text { para distintas configuraciones de robot } \\
\text { (Barrientos, 1997). } \\
\text { Construcción de un software para simular la } \\
\mathrm{Cl} \text { con animación gráfica, con ingreso de } \\
\text { datos por teclado, para cada una de las } \\
\text { configuraciones de robots propuestas. }\end{array}$ & $\begin{array}{l}\text { Comprender la solución de la } \\
\text { cinemática inversa de un robot. }\end{array}$ \\
\hline $\begin{array}{l}6 \\
\text { Trabajo } \\
\text { final } \\
\text { Integra- } \\
\text { dor }\end{array}$ & $\begin{array}{l}\text { Construcción de un software para simular el } \\
\text { control cinemático, con animación gráfica, } \\
\text { de un robot antropomorfo de tres grado de } \\
\text { libertad (Barrientos, 1997). }\end{array}$ & $\begin{array}{l}\text { Comprender la solución del control } \\
\text { cinemático de un robot. } \\
\text { Integrar todos los contenidos. }\end{array}$ \\
\hline
\end{tabular}

Análisis de las actividades de comprensión

En la construcción de los simuladores el alumno aparte de aplicar el conocimiento específico, debe programar utilizando las ecuaciones obtenidas en el modelo de $\mathrm{D}-\mathrm{H}$, lo cual es una situación más compleja de lo que normalmente se hace, y lo lleva a utilizar el conocimiento más allá de lo común. La realización del programa motiva a los estudiantes porque la actividad es interactiva, inspira su creatividad y representa un desafío que los lleva a generar cada vez programas más sofisticados. 
Hay un software básico que los estudiantes deben realizar y es el que todos logran hacer, pero a partir de este, algunos estudiantes van modificando el programa agregando sofisticaciones orientadas a obtener más información del simulador, como por ejemplo sistemas de ejes coordenados en el extremo del modelo, la restitución automática de la posición del extremo del robot etc. También modifican el aspecto del robot utilizado, generando presentaciones estéticamente más representativas.

En el caso particular del trabajo final integrador donde se debe realizar la simulación del Control Cinemático de un Robot, el simulador obtenido permite analizar, comprender y ejecutar la estrategia de control y ver en la pantalla como se va moviendo cada eje del mismo, obteniendo información gráfica, sobre la posición, velocidad y aceleración de cada articulación en función del tiempo. En este caso el simulador del robot permite alcanzar varios puntos en el espacio en determinados tiempos.

\section{RESULTADOS Y DISCUSIÓN}

Para el análisis y evaluación de las actividades o desempeños de comprensión realizadas por los estudiantes, basado en el marco conceptual de la enseñanza para la comprensión, se utilizó una matriz analítica instruccional conocida como rúbrica, según se muestra en la Tabla 2 (Pogré y Lombarda, 2004). Esta matriz, fue creada específicamente para evaluar las actividades de los alumnos (Morán, 2009). Relaciona las dimensiones y niveles de la comprensión con criterios de evaluación, que a su vez están relacionados con las metas de comprensión planteadas para un tópico generativo particular.

Tabla 2: Matriz o Rúbrica (simplificada)

\begin{tabular}{|l|l|l|l|}
\hline Nimen & \multicolumn{1}{|c|}{$\begin{array}{c}\text { Novato } \\
\text { (bueno) }\end{array}$} & $\begin{array}{c}\text { Aprendiz } \\
\text { (Muy bueno) }\end{array}$ & \multicolumn{1}{|c|}{$\begin{array}{c}\text { Maestría } \\
\text { (Óptimo) }\end{array}$} \\
\hline Contenido & Criterios & Criterios & Criterios \\
\hline Método & Criterios & Criterios & Criterios \\
\hline Propósito & Criterios & Criterios & Criterios \\
\hline Comunicación & Criterios & Criterios & Criterios \\
\hline
\end{tabular}

Por una razón de extensión se presentan los criterios sin desarrollar. A modo de ejemplo solo se desarrollan los correspondientes a la dimensión de los contenidos, para los diferentes niveles.

En la dimensión de los contenidos para el nivel de novato los criterios fueron: El robot simulado solo se posiciona en algunos octantes del espacio. El alumno no salva las indeterminaciones. No presenta alternativas para solucionar los límites impuestos al diseño. No siempre elige el camino más corto entre dos puntos. Las variables de posición, velocidad y aceleración están graficadas fuera de escala, debido a la falta de habilidad con el programa. El movimiento del robot no está perfectamente simulado, presenta saltos cuando pasa por indeterminaciones no salvadas.

En la dimensión de los contenidos para el nivel de aprendiz los criterios fueron: El robot simulado se posiciona correctamente en la mayoría de los octantes del espacio. Salva la mayoría de las indeterminaciones. No presenta alternativas para solucionar los límites impuestos al diseño. Elige el camino más corto entre dos puntos. Las variables de posición, velocidad y aceleración están bien graficadas. El movimiento del robot está perfectamente simulado. No presenta ideas innovadoras.

En la dimensión de los contenidos para el nivel de maestría los criterios fueron: El robot simulado se posiciona correctamente en todos los octantes del espacio: Todas las indeterminaciones están salvadas. Presenta variantes para solucionar los límites impuestos al diseño. Siempre elige el camino más corto entre dos puntos. Las variables de posición, velocidad y aceleración en función 
del tiempo están correctamente graficadas. El movimiento del robot está perfectamente simulado. Presenta ideas innovadoras al nivel de sofisticaciones.

La matriz o rúbrica de la Tabla 2 se aplicó para evaluar los trabajos finales integradores de los alumnos de las cohortes 2007 que cursaron la asignatura robótica. Algunos autores consideran a demás de estos niveles el de ingenuo, pero los alumnos que realizan el trabajo integrador ya han superado ese nivel de comprensión, por lo que no es necesario analizarlo. Cada uno de los trabajos prácticos, parciales y principalmente los trabajos finales integradores fueron recuperados al finalizar el cursado y estuvieron disponibles para realizar el presente trabajo. Particularmente el trabajo integrador debe ser aprobado mediante un informe escrito y luego defendido en forma oral en clase.

Para determinar los niveles de comprensión de cada actividad final integradora, se cuenta con el informe escrito del trabajo que incluye el código del programa, un CD con el simulador y las respuestas obtenidas durante la comunicación oral. También se cuenta con registros del seguimiento que se hace de los trabajos durante las consultas.

De esta manera, examinando toda la información disponible, se determinó para cada alumno, de acuerdo a la performance de sus trabajos, que niveles de comprensión habían alcanzado para las diferentes dimensiones, según los criterios establecidos en la tabla 2, precisando para cada caso, si se correspondía con el nivel de novato (bueno), aprendiz (muy bueno) o de maestría (óptimo). Es importante destacar que en el marco de la enseñanza para la comprensión los criterios de evaluación deben ser públicos y previamente establecidos, por lo que los alumnos tienen acceso a los mismos antes de presentar sus trabajos

En la Tabla 3 se observa que un 7,4\% de los estudiantes alcanzan el nivel de novato. Estos estudiantes solo regularizan la asignatura y deben rendir un examen final. El 63\% que alcanzan el nivel de aprendiz más el 29,6\% que obtienen el nivel de maestría promocionan la asignatura, es decir que la aprueban sin examen final.

Se deduce que las características de las actividades de comprensión a realizar y el nivel exigido para promocionar, aseguran que la mayoría de los estudiantes alcancen como mínimo un nivel de aprendiz (muy bueno), en cada dimensión de la comprensión.

Tabla 3: Resumen de Resultados

\begin{tabular}{|l|c|c|c|c|c|c|}
\hline \multicolumn{1}{|c|}{$\begin{array}{c}\text { Novato } \\
\text { (Bueno) }\end{array}$} & \multicolumn{2}{c|}{$\begin{array}{c}\text { Aprendiz } \\
\text { (Muy Bueno) }\end{array}$} & \multicolumn{2}{c|}{$\begin{array}{c}\text { Maestría } \\
\text { (Óptimo) }\end{array}$} \\
\hline Dimensión & 2 & $7,4 \%$ & 17 & $63 \%$ & 8 & $29,6 \%$ \\
\hline Cotal de alumnos evaluados 27 & 2 & $7,4 \%$ & 17 & $63 \%$ & 8 & $29,6 \%$ \\
\hline Método & 2 & $7,4 \%$ & 17 & $63 \%$ & 8 & $29,6 \%$ \\
\hline Propósito & 2 & $7,4 \%$ & 17 & $63 \%$ & 8 & $29,6 \%$ \\
\hline Comunicación & & & & & &
\end{tabular}

Es notable que los desempeños de los alumnos sea uniforme respecto de las diferentes dimensiones de la comprensión, es decir que aquellos trabajos que en contenido alcanzan un desempeño de un nivel de novato, luego lo mantiene en las otras dimensiones analizadas. Como se puede observar en la tabla 3, lo mismo sucede con los otros niveles

\section{CONCLUSIONES}

Se ha logrado diseñar, elaborar e implementar un dispositivo didáctico innovador basado en la construcción progresiva de un software de simulación, a partir de un lenguaje de programación, que permite completar, favorecer y facilitar la comprensión de los estudiantes. Para ello se 
elaboraron actividades que constituyen un proceso de construcción y apropiación de conocimientos fundamentales sobre los temas abordados.

El uso de la informática para realizar las actividades de comprensión permite perfeccionar y enriquecer los desempeños realizados por los alumnos de diversas maneras. El software construido a partir del modelo y la simulación hace visible conceptos abstractos y permite que los estudiantes comprendan ideas complicadas experimentando activamente con ellas, manipulando variables y observando la interacción dinámica entre los diferentes elementos que componen el programa de simulación del robot.

Un aporte de este trabajo es la construcción de la matriz analítica instruccional tabla 2, ya que en el futuro puede servir de referencia para otros trabajos similares al presente y de esta manera ir enriqueciéndola y perfeccionándola. Esta matriz por otra parte puede ser utilizada para la evaluación de los estudiantes, en cuyo caso es recomendable su difusión pública. Esto induce al alumno a realizar una autoevaluación que mejora el nivel de sus desempeños.

\section{REFERENCIAS}

Alimisis, D., y otros 5 autores; Robotics \& Contructivism in Education: The TERECoP project, Proceedings of the $11^{\text {th }}$ European Logo Conference, Bratislava, Eslovakia, 19 a 24 de Agust (2007), http://www.terecop.eu/downloads/P-Alimisis.pdf.

Barrientos, A., y otros 3 autores; Fundamentos de Robótica, 1era edición, 93-112, 169-184, McGraw-Hill, Madrid, España (1997).

Blythe, T.; La Enseñanza para la Comprensión: Guía para el docente, 3era edición. 43-127, Paidos, Buenos Aires, Argentina (1999).

Bravo, C.A.J., y otros 2 autores; Laboratorios virtuales y remotos basados en EJS para la enseñanza de robótica industrial, XXVIII Jornadas de Automática, Huelva, España, 5 a 7 de septiembre

http://rua.ua.es/dspace/bitstream/10045/2230/1/LABORATORIOS_VIRTUALES.pdf

(2007),

Herías, F.A.C., y otros 2 autores; Flexible Virtual and Remote Laboratory for Teaching Robotics, IV International Conference on Multimedia and Information and Comunication Technologies in Education, Sevilla, España, 22 a 25 de Noviembre (2006), http://www.formatex.org/micte2006/pdf/19591963.pdf

Moran, O.D.; Enseñanza para la Comprensión en Robótica - Tópicos que implican movimientos controlados numéricamente, Tesis de Magister. Universidad Nacional de San Luis, Facultad de Ciencias Humanas, San Luis, Argentina (2009)

Pogré, P., y G. Lombarda; Escuelas que Enseñan a Pensar: Enseñanza para la comprensión un marco teórico para la acción, 1era edición, 94-98, Educación papers editores, Buenos Aires, Argentina (2004).

Rawat, K.S., y G.H. Massiha; Model Driven Robot Simulation: RoboCell, Proceedings of the 2003 ASSE Gulf - Southwest anual conference the University of Texas at Arlignston, USA (2003), http://www.aseegsw.org/Proceedings/ASEE-GSW-raw1.pdf.

Wiske, M. S., y otros 2 autores; Enseñar para la Comprensión: Con nuevas tecnologías, 1era edición, 67-183, Paidos, Buenos Aires, Argentina (2006). 